\title{
Diseño de una plataforma móvil en dos dimensiones con resolución nanométrica en un amplio rango de trabajo
}

\author{
Margarita Valenzuela, Marta Torralba, José Antonio Yagüe, José Antonio Albajez, \\ Juan José Aguilar
}

GIFMA (Grupo de Ingeniería de Fabricación y Metrología Avanzada)

Instituto de Investigación en Ingeniería de Aragón (I3A).

Universidad de Zaragoza, Mariano Esquillor s/n, 50018, Zaragoza, Spain.

Tel. +34-976762707, Fax +34-976762043, e-mail: margarita.vgm@gmail.com

\begin{abstract}
En este trabajo se presenta el diseño inicial de una plataforma de desplazamiento en dos dimensiones en un rango de $50 \mathrm{~mm}$ x $50 \mathrm{~mm}$. Se explican los componentes utilizados, como materiales, sensores, accionamientos, etc. para lograr resoluciones nanométricas y precisiones submicrométricas, así como las pruebas realizadas a los mismos.
\end{abstract}

\section{Introducción}

En los últimos años la demanda de micro y nanocomponentes se ha incrementado notablemente, lo que ha aumentado la necesidad de sistemas de fabricación y medición para dichos componentes. En concreto, para que estos sistemas de medición alcancen las altas prestaciones necesarias, es fundamental la utilización de dispositivos de posicionamiento extremadamente precisos.

Aunque existe una gran variedad de posicionadoras que alcanzan las precisiones y resoluciones necesarias para su uso (litografía, microscopios, etc.), sus rangos de medición suelen ser muy limitados. Esta restricción puede ser un factor clave en aplicaciones de tratamiento de muestras de grandes dimensiones con estructuras a micro escala, como vidrios, láminas de silicio, papel, ópticas, etc.

Dada el área de oportunidad de nanoposicionadoras con amplios rangos, en este trabajo se presenta el diseño de una plataforma móvil 2D (en adelante "plataforma") que puede alcanzar en un amplio rango de movimiento resoluciones nanométicas, para utilizarse en el campo de la nanotecnología no cubierta por otras nano-posicionadoras.

\section{Especificaciones de la plataforma}

El diseño de la plataforma se basa en cubrir un rango de $50 \mathrm{~mm}$ x $50 \mathrm{~mm}$, con resolución y estabilidad de $1 \mathrm{~nm}$, precisión de $10 \mathrm{~nm}$ y una velocidad de $2 \mathrm{~mm} / \mathrm{s}$, así como en los principios básicos del diseño de precisión: minimización de los errores de Abbe y las fuerzas de fricción, utilización de marcos metrológicos, etc [1].

La estructura de la plataforma será coplanar (en un mismo plano los ejes de movimientos en $\mathrm{X}$ e $\mathrm{Y}$ ), en vez de superpuesta (ejes apilados), ya que con esta estructura es posible minimizar al máximo los errores de Abbe en dirección vertical, los errores geométricos en el movimiento causados por movimientos parásitos del eje anterior, desalinamientos y errores de perpendicularidad.

La plataforma constará de tres partes principales: parte fija inferior "base", parte móvil "plataforma móvil” y una parte fija superior "tapa”. En la base y en la plataforma móvil se implementará un marco metrológico para asegurar el cumplimiento de los requisitos de precisión y minimizar influencias térmicas. La plataforma móvil se desplazará en dos dimensiones, sin embargo se medirán sus seis grados de libertad para corregir posibles desviaciones.

\section{Componentes}

En el desarrollo de la plataforma se ha buscado integrar en la medida de lo posible componentes comerciales. En la Figura 1 se muestra el diseño con todos los componentes a utilizar.

Para medir el desplazamiento en el plano XY y el giro de ese plano con respecto a $\mathrm{Z}$ se utilizarán 3 interferómetros láser de doble paso de Renishaw (RLE10) con $1 \mathrm{~nm}$ de resolución. Para determinar los errores de rectitud, errores de cabeceo y vuelco de la plataforma móvil se utilizarán tres sensores capacitivos de Lion Precision (C5-E Elite Driver) con resolución nanométrica.

La plataforma móvil debe contar con un sistema de levitación para obtener un movimiento sin fricción con respecto a la base. Dicha sustentación se 
realizará por un sistema neumático, formado por tres cojinetes de aire con precarga de vacío (VPL 50 mm diameter-S205001), los cuales además de proporcionar una capa de aire entre la base y la plataforma móvil, proveerán de una mayor rigidez a la misma.



Figura 1. Diseño de la plataforma.

Para los marcos metrológicos se usará Zerodur, que es un material de muy bajo coeficiente de dilatación térmica, debido a que no será del todo posible conseguir unas condiciones ambientales totalmente controladas (temperatura de $20^{\circ} \mathrm{C} \pm 0,1^{\circ} \mathrm{C}$ ). Para el resto de los elementos estructurales se propuso el uso de aluminio por sus buenas características mecánicas, capacidad de disipar el calor y bajo coste, en comparación con los materiales de bajo coeficiente de dilatación térmica.

El desplazamiento de la plataforma móvil se realizará con cuatro motores lineales planos Halbach de tres fases (fabricados a medida), que generarán dos fuerzas, una horizontal en la dirección de desplazamiento y otra vertical perpendicular a la superficie del camino magnético del mismo motor. Con ellos se lograría un desplazamiento sin rozamiento, sin grandes fuerzas de atracción y en un rango amplio de trabajo.

Por último, se integrará un AFM, ya que esta será una de las posibles aplicaciones de la plataforma dentro del campo de la nanotecnología. El cabezal del AFM será solidario a la plataforma móvil y las muestras podrán ser escaneadas mediante un nanoposicionador comercial (rango de aproximadamente $100 \mu \mathrm{m}$ x $100 \mu \mathrm{m}$ x $25 \mu \mathrm{m}$ ) solidario a la base.

\section{Ensayos de componentes}

Se realizaron varias pruebas para caracterizar tanto los sensores capacitivos junto con los cojinetes de aire (Figura 2.a), así como el sistema láser junto con el material de Zerodur y un sensor 2D (Figura 2.b). Los resultados de los cojinetes de aire muestran un perfecto comportamiento de estos a una carga igual a la que tendrá la posicionadora (Figura 3.a). En la Figura 3.b se observan errores obtenidos de un ensayo a $20^{\circ} \mathrm{C}$ del sistema laser.
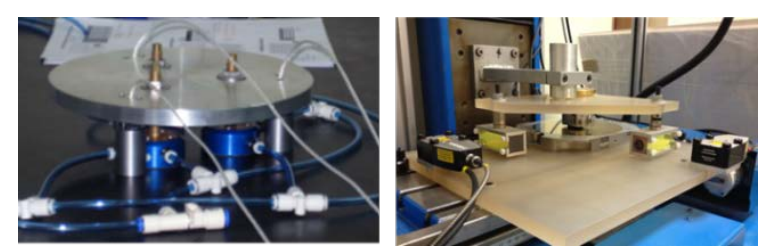

Figura 2. Ensayos: a) sensores capacitivos y cojinetes de aire; b) sistema láser y Zerodur.


Figura 3. Resultados: a) cojinetes de aire; b) Mapa de errores del ensayo del sistema láser, amplificados por un factor $=3000$.

\section{Conclusiones}

Se presentó el análisis y ensayos de los posibles componentes del diseño de una plataforma móvil en dos dimensiones con amplio rango de movimiento, la cual puede ser utilizada en el campo de la nanotecnología.

\section{Acknowledgement}

Trabajo financiado por el gobierno Español a travez del proyecto: DPI2010-21629-C02-01 “NanoPla”. Agradecimiento a CONACYT y DGEST por financiar la primera autora.

\section{Referencias}

Schellekens, P. and Rosielle, N. 1998. Design for precision: Current status and trends. Annals of the CIRP 47, 557-58. 